\title{
Temperature Dependence Of Energy States And Band Gap Broadening
}

\section{${ }^{1}$ Sharibayev Nosirjon Yusufjanovich, ${ }^{2}$ Dadamirzaev Muzaffar Gulomkodirovich, ${ }^{3}$ Sharibaev Raximjon Nosirjon O'g'li}

${ }^{1}$ Doctor of Physical and Mathematical Sciences (DSc) of Namangan Engineering-Technological Institute, Namangan, Uzbekistan.

${ }^{2}$ Senior Lecturer of Namangan Civil Engineering Institute, Namangan, Uzbekistan.

${ }^{2}$ Student of f Namangan Engineering-Technological Institute, Namangan, Uzbekistan.

E-mail: sharibayev_niti@mail.ru

Article History: Received:11 January 2021; Accepted: 27 February 2021; Published online: 5 April 2021

\begin{abstract}
Statistical analysis of energy levels is carried out. The density of surface states of MIS structures based on silicon is investigated. A mathematical model is constructed for the temperature dependence of the spectrum of the density of surface states for a wide energy range. A formula is derived for the density of surface states as a function of temperature. The thermal contributions of the expanded bands to the band gap of the semiconductor are taken into account. The resulting formula allows one to determine the density of energy states in the forbidden band in an explicit form, without taking into account the influence of the broadening of the allowed bands. This improves the accuracy of determining the concentration of impurities and defects in silicon.
\end{abstract}

Key words: semiconductor, density of surface states, mathematical model, density of energy states of generalized bands, model of energy bands, temperature dependence, thermodynamic density of states;

\section{Introduction}

When modeling the electrical properties of MIS structures and devices based on them, it is assumed that the do pant is uniformly distributed. However, such an approximation is not always correct, since the redistribution of impurity atoms is possible as a result of various energetic effects on semiconductor structures during their formation. In addition, the localized states can be affected by the thermal broadening of the allowed bands, which are estimated at room temperature. Thus, taking into account the well-known distribution law of electrically active impurities when calculating the density of surface states is an urgent task and will make it possible to predict the parameters and characteristics of semiconductor devices based on MIS structures. A certain spectrum of the density of surface states carries information about the energy location of the impurity level in the band gap of the semiconductor. [1]due to tail fusion wider energy levels of permitted zones and temperature wider the formation of localized levels.

Modeling of the temperature dependence of the density of surface states for a wide energy range, which includes the allowed and forbidden bands of a semiconductor, it becomes possible to obtain more accurate information on the nature of localized states at the semiconductor - [2].

Temperature-dependent delta function to determine the energy level

The resulting function,

$$
\rho(t)=1-\exp \left[-\frac{t}{\tau(E)}\right]
$$

explain the ideal model describing the process of ionization from the energy level E0 [1]. The model is as follows (Fig. 1): during time $\mathrm{t}$ all electrons with energies above E1 were generated in the ZP, the rest of the electrons with energies below E1 remain in their places.

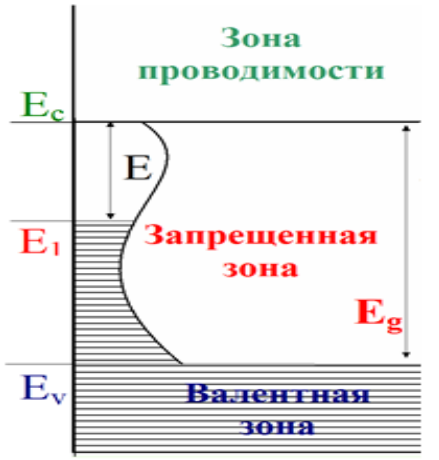

Fig. 1. Ideal model representation 
By this, formula (1) of the ideal model is a step function. In work [2,3] $\tau(E)$ as determining the lifetime of an electron which is strongly dependent on the energy $E$ is represented in the form

$$
\tau(E)=\tau_{0} \exp \left(\frac{E}{\kappa T}\right)
$$

Here: $\tau_{0}$ electron lifetime, k-Boltzmann constant, $\mathrm{T}$-absolute temperature.

As a result, the following expression was obtained from (1.22) and (2.1):

$$
\rho(t, E, T)=1-\exp \left[-\exp \left(\frac{k T \ln \left(\frac{t}{\tau_{0}}\right)-E}{k T}\right)\right]
$$

In this formula, the expression $k T \ln \left(\frac{t}{\tau_{0}}\right)$ represents energy. Let us denote it by the new variable E0.

$$
E_{0}(t, T)=k T \ln \left(\frac{t}{\tau_{0}}\right)
$$

(4) represents the energy of an electron, which electrons with energies greater than E0 have been ionized. In this expression, we note the peculiarity that E0 depends on the temperature and on the generation time. If we consider time as an ideal parameter, then it will be possible for each value of the [3]. In other words, (4) can be considered as

$$
E_{0}=k T \ln \left(\frac{t}{\tau_{0}}\right)
$$

Now formula (3) can be represented as

$$
\rho\left(E_{0}, E, T\right)=1-\exp \left[-\exp \left(\frac{E_{0}-E}{k T}\right)\right]
$$

Where $E_{0}$ is the electron energy independent of temperature, which electrons with energies greater than $E_{0}$ were ionized, E-electron energy, which levels with energies greater than E0 filled (Fig. 1), k-Boltzmann constant, Tabsolute temperature. [4]

The mathematically derivative of a step function with respect to its argument gives the delta function. The well-known in statistical physics the Dirac Delta-function determines the presence of an energy level [4]. In the same way, the derivative of function (6) with respect to energy E0

$$
\delta^{*}\left(E_{0}, E, T\right)=\frac{\partial \rho\left(E_{0}, E, T\right)}{\partial E_{0}}=\frac{1}{k T} \exp \left(\frac{1}{k T}\left(E-E_{0}\right)-\exp \left(\frac{1}{k T}\left(E-E_{0}\right)\right)\right)
$$

is a delta function for $T \rightarrow 0$ [2,3,5] and determines the presence of a level from which an electron with energy E0 can be ionized or the presence of a level with energy E at which an [5]. Delta functions (7) at different temperatures are shown in Fig. 2. 


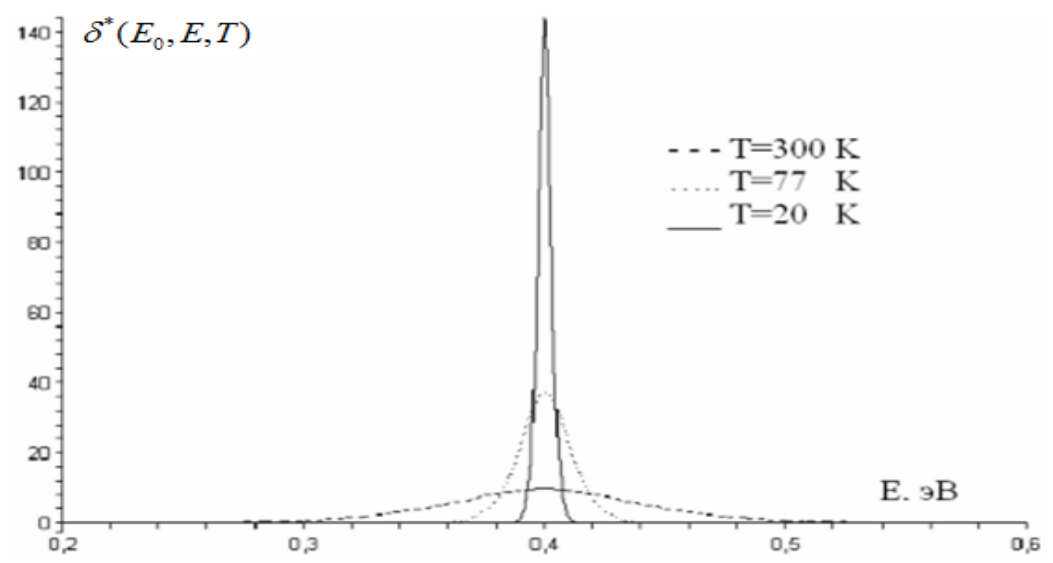

Fig. 2. Delta functions $\delta^{*}\left(E_{0}, E, T\right)$ at different temperatures

\section{Materials and Methods}

Model of the thermodynamic density of surface states

On the surface, the following expressions are usually used to determine the concentration of electrons at the edges of the bands:

$$
\begin{aligned}
& n_{s}\left(E_{f}, T\right)=2 \int_{E_{c}}^{\infty} N_{c}\left(E-E_{c}\right)^{\frac{1}{2}} f\left(E, E_{f}, T\right) d E \\
& p_{s}\left(E_{f}, T\right)=2 \int_{-\infty}^{E_{v}} N_{p}\left(E_{v}-E\right)^{\frac{1}{2}} f\left(E, E_{f}, T\right) d E
\end{aligned}
$$

where the Fermi - Dirac function $f(E, T)$ determining the probability that the electron will be in a quantum state with energy $\mathrm{E}$ is [6]:

$$
f\left(E, E_{f}, T\right)=\frac{1}{1+\exp \left(\frac{E-E_{f}}{k T}\right)}
$$

As is known, when calculating integral (11), as a result of the change of variables, the integrand is transformed into a delta function, the effective density of states is transformed into $N_{c}=\left(\frac{2 \pi m_{p} k T}{h^{2}}\right)$, $N_{v}=\left(\frac{2 \pi m_{v} k T}{h^{2}}\right)$ and in this case the integral is equal to one.[7] But function (6) differentiated with respect to $E_{0}$ also becomes $\delta$ is a function and therefore in (10) and (11) one can replace (12) by function (6). Then the expressions for the concentrations will take the following form:

$$
\begin{aligned}
& n\left(E_{0}, T\right)=2 \int_{E_{c}}^{\infty} N_{0_{c}}\left(E-E_{c}\right)^{\frac{1}{2}} \rho\left(E_{0}, E, T\right) d E \\
& p\left(E_{0}, T\right)=2 \int_{-\infty}^{E_{v}} N_{0_{p}}\left(E_{v}-E\right)^{\frac{1}{2}} \rho\left(E_{0}, E, T\right) d E
\end{aligned}
$$

Where, for the surface $N_{0_{c}}=4 \pi\left(\frac{2 m_{n}^{*}}{h^{2}}\right), \quad N_{0 v}=4 \pi\left(\frac{2 m_{p}^{*}}{h^{2}}\right)$

Here, the parameter E0, determined from (4), can be called an energy parameter of the Fermi energy type, which now depends on time and temperature. Formula (5), as well as (12), determines the probability of filling the level with energy $\mathrm{E}$, but this probability depends in this case also on the value $E_{0} \ldots$ The parameter $\mathrm{E}_{0}$ depends on the T-temperature and t-time variables[8]. And when selecting for a given $\mathrm{T}$, corresponding to $\mathrm{t}$, the 
value of E0 becomes, as it were, independent of the temperature and becomes, in terms of this independent variable, it is possible to very simply differentiate (11) and (12):

$$
\begin{aligned}
& \frac{\partial n\left(E_{0}, T\right)}{\partial E_{0}}=2 \cdot N_{c} \cdot \int_{E_{c}}^{\infty}\left(E-E_{c}\right)^{\frac{1}{2}} \frac{\partial \rho\left(E_{0}, E, T\right)}{\partial E_{0}} \\
& \frac{\partial p\left(E_{0}, T\right)}{\partial E_{0}}=2 \cdot N_{\nu} \cdot \int_{-\infty}^{E_{v}}\left(E_{v}-E\right)^{\frac{1}{2}} \frac{\partial \rho\left(E_{0}, E, T\right)}{\partial E_{0}}
\end{aligned}
$$

Here is the integrand depending on $E_{0}$ in (7) is defined by a function that for $T \rightarrow 0$ becomes $\delta$-function. Then the formula for [9] of states in the conduction and valence bands will have the following form:

$$
\begin{aligned}
& N s_{c}\left(E_{0}, T\right)=2 \cdot N_{0_{c}} \cdot \int_{E_{c}}^{\infty}\left(E-E_{c}\right)^{\frac{1}{2}} \delta^{*}\left(E_{0}, E, T\right) d E \\
& N s_{v}\left(E_{0}, T\right)=2 \cdot N_{0_{v}} \cdot \int_{-\infty}^{E_{v}}\left(E_{v}-E\right)^{\frac{1}{2}} \delta^{*}\left(E_{0}, E, T\right) d E,
\end{aligned}
$$

Generalizing formulas (16), (17) and taking into account the fact that there are no energy levels in the band gap, we can write a generalized expression for the combined density of states for the valence band, band gap and conduction band[10].

$$
N_{s s}\left(E_{0}, T\right)=2 \cdot N_{0_{v}} \cdot \int_{-\infty}^{E_{v}}(-E)^{\frac{1}{2}} \delta^{*}\left(E_{0}, E, T\right) d E+2 \cdot N_{0_{c}} \cdot \int_{E_{c}}^{\infty}\left(E-E_{g}\right)^{\frac{1}{2}} \delta^{*}\left(E_{0}, E, T\right) d E
$$

Figure 4 shows the righteous graphs (18) for different temperatures. The figure shows how the formula takes into account the temperature broadening of the energy bands.

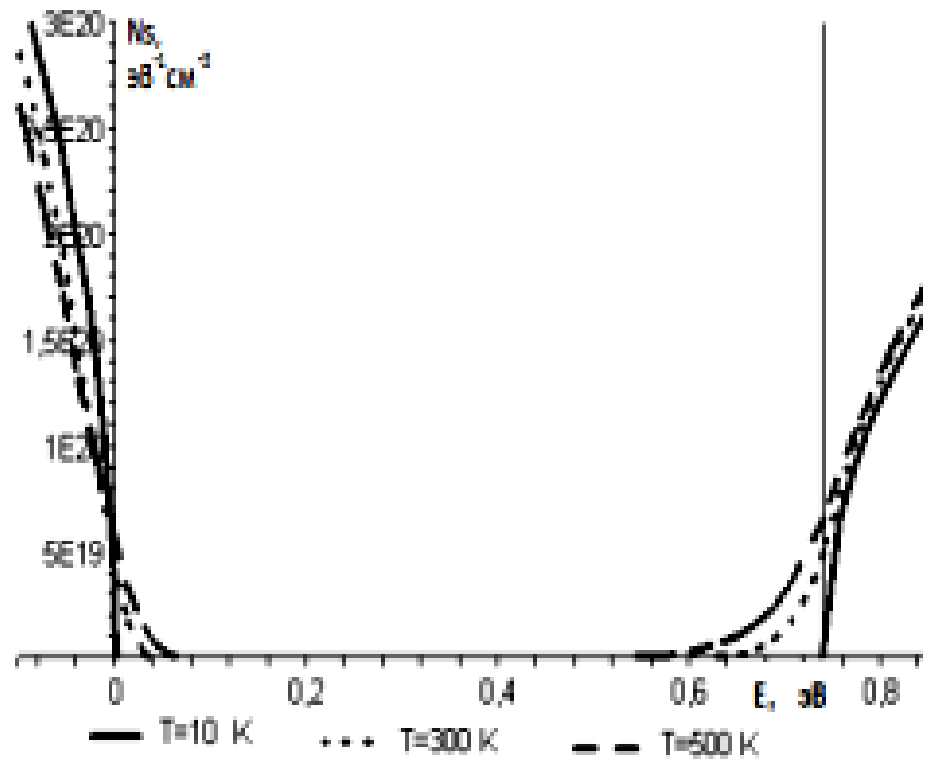

Figure: 3 (Figure 3).graphs (18) for different temperatures

PPP formula for generalized energy bands (Results)

The resulting integral formula (eighteen)difficult to compute analytically. For convenience, we will use an approximation[11]. To begin with, in (7) the exponential function in the exponent to the second power and we get the following:

$$
\delta_{1}^{*}\left(E_{0}, E, T\right)=\frac{e^{-1}}{k T} \exp \left(-\left(\frac{E-E_{0}}{\sqrt{2} k T}\right)^{2}\right)
$$

Subsequent approximation we get:

$$
\delta_{g}\left(E_{0}, E, T\right)=\frac{e^{-1}}{k T}\left(1+\left(\frac{E-E_{0}}{\sqrt{2} k T}\right)^{2}\right)^{-1}
$$




$$
N s_{v}\left(E_{0}, T\right)=2 \cdot N_{0_{v}} \cdot \int_{-\infty}^{E_{v}}(-E)^{\frac{1}{2}} \delta_{g}\left(E_{0}, E, T\right) d E
$$

Now we can calculate the integral for the valence band using the analytical method. After calculations, we get the following expression:

$$
N s_{v}\left(E_{0}, T\right)=\frac{\sqrt{2} N_{0_{v}} \pi k T e^{-1}}{\sqrt{\sqrt{2 k^{2} T^{2}+E_{0}^{2}}+E_{0}}}=\pi e^{-1} N_{0_{v}} \sqrt{\sqrt{2 k^{2} T^{2}+E_{0}^{2}}-E_{0}}
$$

Similarly for the conduction band

$$
\begin{gathered}
N s_{c}\left(E_{0}, T\right)=\frac{\sqrt{2} N_{0_{c}} \pi k T e^{-1}}{\sqrt{\sqrt{2 k^{2} T^{2}+\left(E_{0}-E_{g}\right)^{2}}+\left(E_{0}-E_{g}\right)}}=\pi e^{-1} N_{0_{c}} \sqrt{\sqrt{2 k^{2} T^{2}+\left(E_{0}-E_{g}\right)^{2}}-\left(E_{0}-E_{g}\right)} \\
\text { Where } N_{0_{c}}=\frac{8 \pi m_{n}^{*}}{h^{2}}, \quad N_{0_{v}}=\frac{8 \pi m_{p}^{*}}{h^{2}} \\
N s s\left(E_{0}, T\right)=2 \pi^{2} h^{-2} e^{-1}\left(m_{v}^{*} \sqrt{\sqrt{2 k^{2} T^{2}+E_{0}^{2}}-E_{0}}+m_{c}^{*} \sqrt{\sqrt{2 k^{2} T^{2}+\left(E_{0}-E_{g}\right)^{2}}-\left(E_{0}-E_{g}\right)}\right)
\end{gathered}
$$$$
\text { When } m_{0}=m_{v}=m_{c}
$$$$
N s s\left(E_{0}, T\right)=2 \pi^{2} m_{0} h^{-2} e^{-1}\left(\sqrt{\sqrt{2 k^{2} T^{2}+E_{0}^{2}}-E_{0}}+\sqrt{\sqrt{2 k^{2} T^{2}+\left(E_{0}-E_{g}\right)^{2}}-\left(E_{0}-E_{g}\right)}\right)
$$

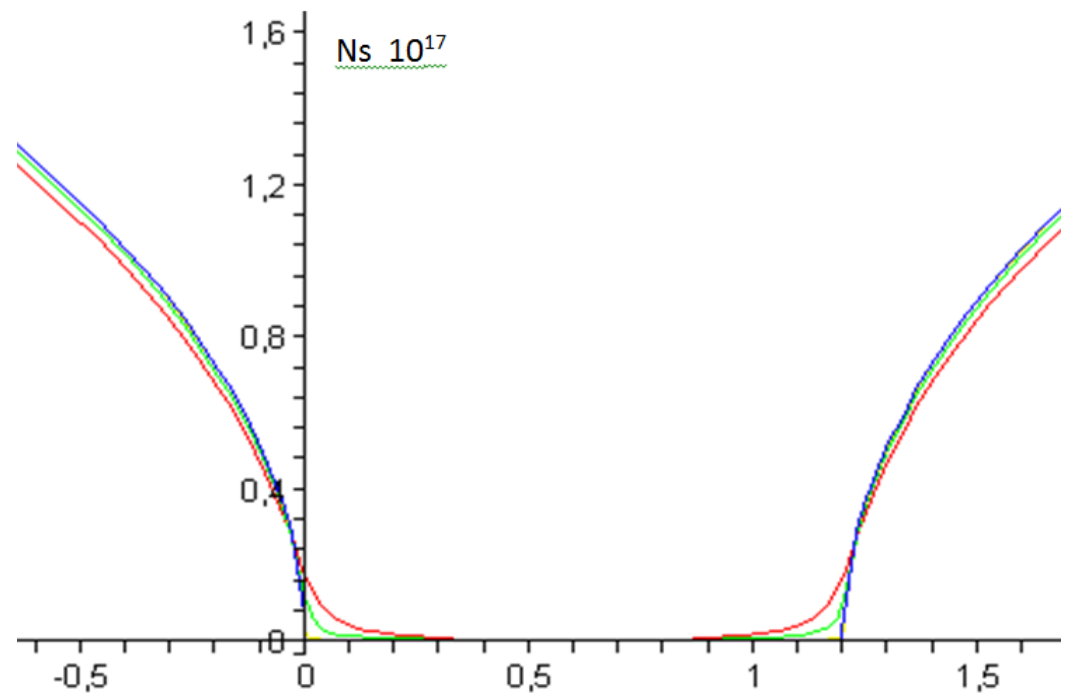

Using formula (23), one can calculate the density of energy states in a wide energy range, including the valence band, the forbidden band, and the conduction band. A feature of the model is the preservation of the temperature dependence of statistical functions, which help determine the contribution of thermal broadening to the spectrum of the density of energy states[11]. The proposed method allows one to determine discrete energy levels created by impurities with greater accuracy over a wide energy range.[12]

\section{Results and Discussions}

The PS model has been studied in many literatures. In the particular case of an isotropic quadratic dispersion law, the density of energy states for energies lower than E can be determined as follows [6-9]:

$$
\begin{aligned}
& N s_{c}(E)=\frac{1}{4 \pi^{2}}\left(\frac{2 m_{c}^{*}}{\hbar^{2}}\right)\left(E-E_{c}\right)^{1 / 2} \\
& N s_{v}(E)=\frac{1}{4 \pi^{2}}\left(\frac{2 m_{v}^{*}}{\hbar^{2}}\right)\left(E_{v}-E\right)^{1 / 2}
\end{aligned}
$$


This expression does not take into account the temperature broadening of the energy levels. Formula (25) and (26) are obtained from (11) and (12) due to the property of the delta function. Because $\int_{-\infty}^{\infty} \delta\left(E-E_{0}\right) d E=1 \ldots$ In the resulting model, this is achieved when $T \rightarrow 0$, where (7) and (19) become delta functions. To take into account the temperature dependence of the TS, we use (25) and (26). Then we get the spectrum of the density of energy states depending on temperature.[13] Fig. 6 (a, b, c, d) shows comparative spectra of PS in the valence band $(a, b)$ and in the ZP (c, d) at low and high temperatures.

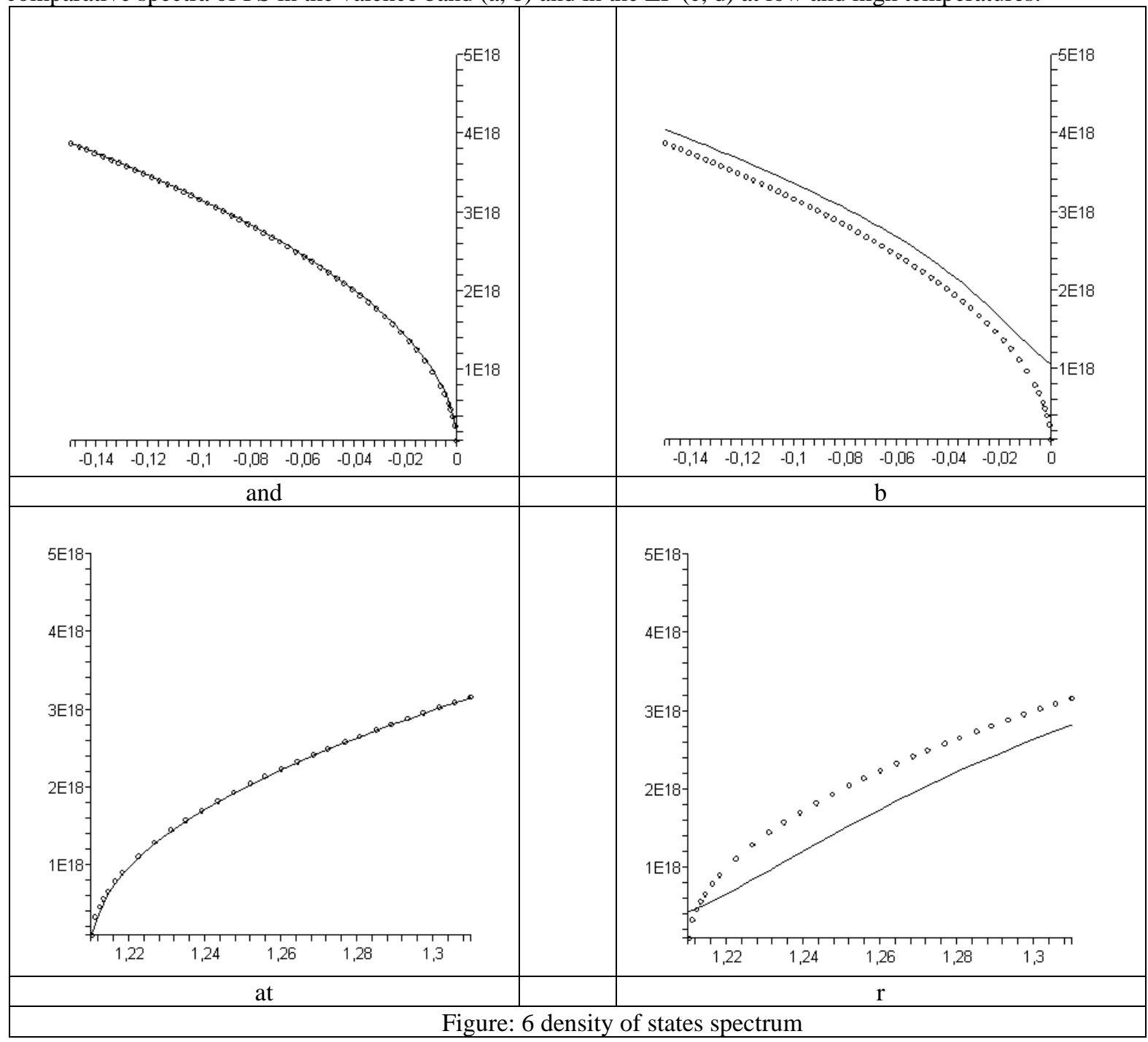

Comparisons show that the developed model describes the spectrum of the density of energy states at temperatures close to absolute zero, where the lines coincide. This is because at low temperatures function (7) and (19) turns into a delta function [14].

The well-known Kane model is often used to describe non-parabolic zone types [10-11]:

For the permitted zones separately and we get for the air intake: $E<0$

$$
N_{s V}(E)=\frac{\left(m_{n}\right)^{3 / 2}}{2^{1 / 2} \pi^{2} \hbar^{3}}(0-E)^{1 / 2}\left(1+\frac{0-E}{E_{g}}\right)^{1 / 2}\left[1+2\left(\frac{0-E}{E_{g}}\right)-v\left(\frac{0-E}{E_{g}}\right)\left(5+8\left(\frac{0-E}{E_{g}}\right)\right)\right]
$$

and for the RFP: $E>E g$

$$
N_{s V}(E)=\frac{\left(m_{n}\right)^{3 / 2}}{2^{1 / 2} \pi^{2} \hbar^{3}}(E-E g)^{1 / 2}\left(1+\frac{E-E g}{E_{g}}\right)^{1 / 2}\left[1+2\left(\frac{E-E g}{E_{g}}\right)-v\left(\frac{E-E g}{E_{g}}\right)\left(5+8\left(\frac{E-E g}{E_{g}}\right)\right)\right]
$$


Model Kane for the indicated semiconductors where $\mathrm{Eg}<0.5 \mathrm{eV}$ describes a nonparabolic spectrum and for wide-gap semiconductors where $\mathrm{Eg}>2.5 \mathrm{eV}$ describes a parabolic spectrum. The developed model (23) and (24) show the full spectrum of the density of energy states. Figure 7 (a, b) shows comparative spectra of the density of energy states in the valence band $(a, b)$ for non-parabolic and in the ZP $(c, d)$ for non-parabolic types of bands.[15]

Comparisons show that the developed model describes the spectrum of the density of energy states comparable to the Kane model. The superiority of the proposed model lies in the fact that there is

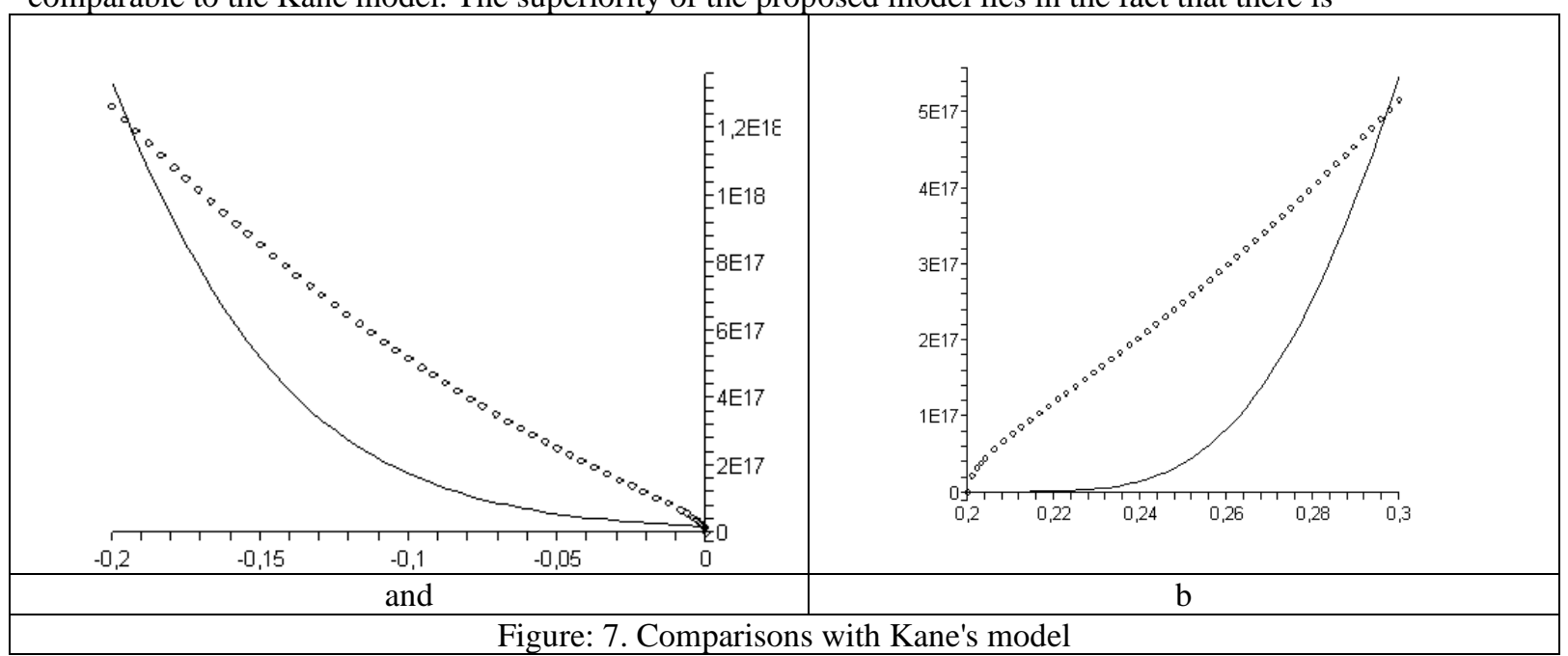

the possibility of taking into account the contribution of temperature action to the spectra of the density of energy states in the ZP and in the valence band The resulting expression (23) is special in that the densities of state can be calculated taking into account the temperature T and the energy of the level E0 and allow one to obtain the spectrum of the temperature dependence of the PS in the ZP, VZ and the forbidden zone.[16]

This is a model to approach a perfectly pure semiconductor where no energy levels exist in the band gap. This formula can be used to calculate the density of energy states, in a wide energy range including the valence band, the forbidden band and the ZP. A feature of the model is the preservation of the temperature dependence of statistical functions. They help to determine the contribution of thermal broadening to the spectrum of the density of energy states. The model can determine the doping contribution to the spectrum change. A high concentration of impurities can significantly change the entire spectrum of the density of energy states[17]. In general, experiments on the determination of impurities are carried out at high temperatures. Determines discrete levels only possible at low temperatures.

\section{Conclusion}

The resulting formula (23) describes the spectrum of the density of the surface state as a function of temperature. Using the formula, it becomes possible to calculate the thermal broadening of the expanded zones. This makes it possible to determine the impurity concentration taking into account the broadening of the expanded bands from the data obtained by the experiment, which was carried out at a high temperature. The resulting model of the density of the surface state takes into account the effect of temperature and is in good agreement with classical theoretical and experimental results. (more than $300 \mathrm{~K}$ ).

\section{References:}

1. S. Nozaki and A. G. Milnes, "Numerical modeling of looped C-V Characteristics in Ap+n junction containing mid-bandgap electron traps," Solid State Electron., vol. 26, no. 2, pp. 115-123, 1983, doi: 10.1016/0038-1101(83)90112-0.

2. D. A. Smolyakov et al., "Influence of metal magnetic state and metal-insulator-semiconductor structure composition on magnetoimpedance effect caused by interface states," Thin Solid Films, vol. 671, pp. 18-21, Feb. 2019, doi: 10.1016/j.tsf.2018.12.026.

3. N. P. Dharmadhikari et al., "Vein width measurement of groundwater on Earth's surface using Semiconductor Laser Light and Proton Precession Magnetometer," J. Appl. Geophys., vol. 171, Dec. 2019, doi: 10.1016/j.jappgeo.2019.103864.

4. G. Gulyamov, U. I. Erkaboev, and N. Y. Sharibaev, "The de Haas-Van Alphen effect at high temperatures and in low magnetic fields in semiconductors," Mod. Phys. Lett. B, vol. 30, no. 7, 2016, doi: 10.1142/S0217984916500779.

5. C. Buono, A. J. Uriz, and C. M. Aldao, "Effects of intergranular capacitance and resistance dispersion on polycrystalline semiconductor impedance," Solid State Ionics, vol. 343, Dec. 2019, doi: 
10.1016/j.ssi.2019.115076.

6. T. Chen, S. Liang, H. Zhang, Y. Sun, S. Yan, and S. Yan, "Non-linear I-V characteristics of a c-axisoriented T12Ba2Ca2Cu3Ox thin film," Solid State Commun., vol. 76, no. 3, pp. 345-347, 1990, doi: 10.1016/0038-1098(90)90851-2.

7. O. D.R, "ENERGY CHARACTERISTICS OF SOLAR PHOTOELECTRIC INSTALLATIONS UNDER THE COMBINED PRODUCTION OF HEAT AND ELECTRIC ENERGY IN THE CONDITIONS OF UZBEKISTAN," Int. J. Psychosoc. Rehabil., 2020, doi: 10.37200/ijpr/v24i4/pr201291.

8. G. Gulyams, M. G. Dadamirzayev, and N. Y. Sharibaev, "INFLUENCE OF REFLECTION OF PHOTOELECTRONS FROM THE SURFACE PHOTO-EMF IN THIN SEMICONDUCTOR FILMS AND COMPARE IT WITH THE BULK PHOTO-EMF," Altern. Energy Ecol., no. 21, 2016, doi: 10.15518/isjaee.2015.21.002.

9. A. Turut, M. Saglam, H. Efeoglu, N. Yalcin, M. Yildirim, and B. Abay, "Interpreting the nonideal reverse bias $\mathrm{C}-\mathrm{V}$ characteristics and importance of the dependence of Schottky barrier height on applied voltage," Phys. B Phys. Condens. Matter, vol. 205, no. 1, pp. 41-50, Jan. 1995, doi: 10.1016/0921-4526(94)00229-О.

10. Ф. Полупроводников, “К . В . Шалимова,” 1985.

11. G. Gulyamov, A. G. Gulyamov, A. Q. Ergashev, and B. T. Abdulazizov, "THE USE OF PHASE PORTRAITS FOR THE STUDY OF THE GENERATION-RECOMBINATION PROCESSES IN SEMICONDUCTORS."

12. G. Gulyamov, N. Y. Sharibaev, and U. I. Erkaboev, "The Temperature Dependence of the Thermodynamic Density of States and Urbach Rule," World J. Condens. Matter Phys., vol. 05, no. 01, 2015, doi: 10.4236/wjcmp.2015.51006.

13. D. R. Otamirzaev, "Experimental Determination of the Efficiency of Solar Panels with Cooling System under the Conditions of Uzbekistan," vol. 29, no. 3, pp. 10680-10686, 2020.

14. G. Gulyamov, U. I. Erkaboev, and N. Y. Sharibaev, "Investigation of the Temperature Dependence of the Oscillation of the Magnetic Susceptibility in Semiconductors," J. Mod. Phys., vol. 05, no. 17, pp. 1974-1979, 2014, doi: 10.4236/jmp.2014.517192.

15. C. Casado, Á. García-Gil, R. van Grieken, and J. Marugán, "Critical role of the light spectrum on the simulation of solar photocatalytic reactors," Appl. Catal. B Environ., vol. 252, pp. 1-9, Sep. 2019, doi: 10.1016/j.apcatb.2019.04.004.

16. W. Barrett et al., "The inverse eigenvalue problem of a graph: Multiplicities and minors," J. Comb. Theory. Ser. B, 2019, doi: 10.1016/j.jctb.2019.10.005.

17. G. Gulyamov, U. I. Erkaboev, and N. Y. Sharibaev, "Simulation of the Temperature Dependence of the Density of States in a Strong Magnetic Field," J. Mod. Phys., vol. 05, no. 08, 2014, doi: 10.4236/jmp.2014.58079. 